\title{
A Single-Arm Clinical Study Assessing Safety and Effectiveness of Path Removal for Delayed Patch Infection After Inguinal Hernioplasty
}

\section{yumin yue}

Department of General Surgery, Shaanxi Provincial People's Hospital

\section{Xiangreng Cai}

Department of General Surgery, Shaanxi Provincial People's Hospital

xin guo

Xi'an Medical University

Xi Chen

Yan'an University

Bobo Zheng ( $\nabla$ zhengbo816@126.com )

Department of General Surgery, Shaanxi Provincial People's Hospital

\section{Research Article}

Keywords: Inguinal Hernia, delayed, patch infection, safety and effectiveness,

Posted Date: February 1st, 2022

DOI: https://doi.org/10.21203/rs.3.rs-1276654/v1

License: (c) (i) This work is licensed under a Creative Commons Attribution 4.0 International License. Read Full License 


\section{Abstract}

Introduction: Patch infection after inguinal hernioplasty as an annoyance perplexed surgeon, Because its processing is very tricky. When conservative treatment fails, the surgical that played a pivotal role in the future therapies. Our target was to evaluate the safety and effectiveness of path removal for delayed patch infection after inguinal hernioplasty and give a summary of our experience in surgical treatment.

Methods: A retrospective cross-sectional study was undertaken at the Department of General Surgery from March 2018 to May 2021 to gather the clinical data of 35 patients with delayed cloth patch infection following tension-free hernioplasty. Record clinical information about the patient which included patient age, underlying disease, primary hernia repair, meantime of infection diagnosis, patient clinical symptoms, the intraoperative complications included the relevant postoperative conditions, the placement and time of drainage tubes, the postoperative hospital stay, the bacterial culture results of patch infection, and the choice and use of antibiotics. The follow-up included postoperative reinfection and long-term complications. Patients or their families were contacted by telephone to understand their postoperative recovery as well as any long-term complications.

Results: All patients have undergone laparoscopic or open surgical operation were smooth and steady. All patients with postoperative infection after tension-free repair of inguinal Hernia received patch removal, all of them were treated with antibiotics, open wound drainage or negative pressure drainage, 2 cases had a perforation of a sigmoid foreign body. The mean postoperative hospital stay was $(23.74 \pm 15.91)$ days. A total of 35 patients with patch infection were followed up postoperatively During the follow-up period, no postoperative complications occurred. There was no recurrence of inguinal hernia during postoperative follow-up.

Conclusion: When dealing with patch infection after tension-free repair of an inguinal hernia, it is safe and efficacious to perform patch extraction.

\section{Introduction}

Inguinal hernia is a common and frequently-occurring disease in surgery. According to statistics, more than 20 million inguinal hernia operations are performed worldwide each year, and the prevalence rate of inguinal hernia in China is $0.3 \%-0.5 \%$, with the elderly over 60 years old with a prevalence rate of up to 5\%. In 1989, "Tension-free Hernia Repair" was proposed by Lichtenstein et Al [1]. This approach is simple and feasible, and it has the potential to significantly reduce postoperative discomfort and recurrence rates. As a result, tension-free hernioplasty has become the mainstream choice for inguinal herniorrhaphy. With the development of herniorrhaphy materials, herniorrhaphy patches are more and more used in various inguinal herniorrhaphy[2-4]. With the extensive use of the patch, there are more and more complications related to patch. Postoperative infection of the patch is one of the more complicated complications. The only way to do that is to surgically remove the infected patch[5-6]. Few other studies show that the incidence of Mesh infection in open tension-free hernioplasty is $6 \%-10 \%$, and has become 
one of the main factors of failure of inguinal hernia surgery[7]. There are still studies [8-9] that showed that certain early patch infections may be treated with conservative treatment, such as wound nursing, empirical antibiotics, and wound negative pressure treatment. However, the majority of patients still fail due to conservative treatment and can lead to recurrent infection of the patch[10]. One of the most challenging complications in postoperative patch infection. The only effective method is the surgical removal of the infected patch[11-12], so once the infection is confirmed, it is often necessary to remove the patch, and may even require multiple operations to remove the infected patch, to achieve satisfactory results. At the same time, many surgeons are still discussing whether to undertake abdominal wall reconstruction once the patch is removed. The focus of this research was to evaluate the safety and effectiveness of path removal for delayed patch infection after inguinal hernioplasty and give a summary of our experience in surgical treatment.

\section{Materials And Methods}

Study population. This retrospective study was performed in department of general surgery, Shaanxi Provincial People's Hospital Xi'an, China; The study protocol adheres to the principles of the Declaration of Helsinki and ethical guidelines that approved by the the Ethics Committee of Shaanxi Provincial People's Hospital. Written informed consent was obtained from all subjects before operation, and ethical approval was obtained.

A retrospective study was conducted, which analyzed data from 35 patients with late-onset reticular infection who were treated in the Department of General Surgery from March 2018 to May 2021. All patients had patch infections before being referred to our hospital and received unsuccessful conservative treatments (such as intravenous empiric antibiotics, percutaneous drainage, and negative pressure wound therapy). All patients have undergone laparoscopic or open surgical operation to remove the infected patch. Before surgery, the routine preoperative examination, Glycated hemoglobin, and inguen CT should be improve and perfect. Patients with incisional sinus formation should have sinus angiography to identify the area of patch infection, all patients were pre-operative incision secretion or aseptic operation under the needle extraction for bacterial culture, and drug sensitivity results of antibiotics, pre-diabetic fasting blood glucose should be controlled in the normal range.

All procedures were carried out under general anesthesia. The previous operation's peritoneum was formed, and the laparoscope was used to examine the inguinal area, except that the patch broke through the peritoneum and entered the abdominal cavity, resulting in infection and entrapment of abdominal viscera, especially the Intestinal Fistula. If there is omentum or intestinal adhesions in the inguinal area, the adhesions should be carefully separated to eliminate intestinal fistula and avoid visceral injury during debridement. If an Intestinal Fistula is found, repair or anastomosis is performed under intraoperative conditions. The operative sequence of the laparoscopy was (1) insertion of a laparoscopic instrument to explore the adhesions between the greater omentum and the patch in the inguinal region under laparoscopy; (2) incision of the peritoneum along the original incision; (3) blunt dissection of the peritoneum to isolate scar adhesions in the gap between the peritoneum and the surrounding tissue; (4) 
found the patch, obtuse separation, removed the infected patch and ensure the integrity of the patch, while protecting protect the bladder, blood vessels and Vas deferens; (5) checked for intestinal Fistula and bladder injury; (6) checked for sinus formation, if marked solution (methylene blue) was injected into the Sinus, complete resection of the sinus; (7) rinsed the abdominal cavity carefully until the water was clear; (8) if the patch was placed the original TAPP space in the inguinal region was free, taken the biological patch, placed the TAPP space in the abdominal wall and pave it, completed coverage of the Muscle Pubic foramen and medial pubic tubercle, fixed the patch with a screw gun, use of absorbable thread to reinforce the suture biomembrane and Abdominal Wall; (9) placement of a negative pressure drainage tube. The operative sequence of the open surgical operation was: A shuttle-shaped incision in the inguinal area is made, and methylene blue or methylene blue is injected into the Sinus Ostium to stain the infected Mesh, sutures, biofilms, and senescent tissue. The surrounding tissue was incised, the patch was located, the sinus was carefully separated, the patch was removed, and the fistula was anastomosed while bowel resection or bladder repair was performed. Carefully protect the spermatic cord, bladder, blood vessels, and other important structures. After debridement, the abdominal aponeurosis was closed with absorbable PDS suture, and a negative pressure drainage tube was placed in the free residual cavity.

Sensitive antibiotics were used according to the results of drainage culture after the operation. The period of antibiotic use was varied based on the patient's entire body and incisions. For patients with diabetes should be timely monitoring of Blood Glucose, Blood Glucose control in the normal range. After the operation, change the dressing regularly, observe the quantity and character of the drainage tube every day, also can carry on the bacterial culture to the drainage fluid. When the drainage is not free, ultrasound inspection is possible to detect whether the drainage tube or double casing core plug occurs, if the plugin time to dredge, and ultrasound site to suck out the fluid accumulation. Extubation should be considered when the postoperative drainage fluid is less than $5 \mathrm{ml}$. When the incision has not graded a healing, double cannulas can be flushed continuously. When a wound infection is severe, it is possible to open the incision and change the dressing several times. Sutures are usually removed in 10-14 d intervals. After three months, pay close attention to coughing, urgent urination, lifting heavy objects, and so on. Postoperative patients must wear the abdominal band for lower activities to prevent intra-abdominal pressure is too high.

Our final observation index was Patient's sex, age, occurrence time of patch infection, clinical manifestations of patch infection, treatment method, operation time, whether to place patch again during operation, whether to place drainage tube during operation, time to remove drainage tube after the operation, type of bacterial culture of drainage fluid, days in the hospital, follow-up. For follow-up, telephone calls were used. The follow-up included the postoperative healing and recurrence of Hernia. The measurement data of normal distribution was expressed by $\mathrm{x} \pm \mathrm{S}$.

\section{Results}

The demographics and preoperative status of 35 patients are presented in Table1, including 27 males and 8 females. Mean patient age was $55.83 \pm 17.35$ years. Of the 35 patients, 2 had diabetes. The mean 
time of infection diagnosis in 35 patients was 31.42 months. Among the preoperative symptoms, there were 28 cases of redness and swelling, 28 cases of pain, 26 cases of Pus, 16 cases of sinus formation, 1 case of change of stool habit, 2 cases of perforation of sigmoid foreign body, 2 cases of abdominal discomfort, 1 case of Hernia, 1 case of mucinous Hematochezia .

Table 1

Baseline characteristics of the included patients

\begin{tabular}{|ll|}
\hline Number & \\
\hline Gender & 27 \\
Male & 8 \\
Female & \\
\hline Age(year) & $58.77 \pm 17.35$ \\
\hline Diabetes & 2 \\
Yes & 33 \\
No & \\
\hline The first hernia operation & \\
\hline Open surgery & 10 \\
\hline laparoscopy surgery & 25 \\
\hline Meantime to diagnosis of infection(month) & 5.67 \\
\hline Clinical symptoms of the patient & \\
\hline Redness and swelling & 28 \\
\hline Pain & 28 \\
\hline Pus & 26 \\
\hline Sinus formation & 16 \\
\hline Change in bowel habits & 1 \\
\hline Perforation of sigmoid foreign body & 2 \\
\hline Abdominal Discomfort & 1 \\
\hline Herniated mass & \\
\hline Mucous, bloody stool & \\
\hline
\end{tabular}

Table2 sum up operative details. The intraoperative conditions of 35 patients were discussed. Among the 35 patients with mesh infection, There are all patients received mesh removal to treat the infection. None of the 35 patients who underwent infection mesh removal had intraoperative complications. Among the 
35 patients undergoing infection mesh removal, 15 patients could not obtain the intraoperative bleeding of the other patients, the average intraoperative bleeding was $10.88 \pm 4.65 \mathrm{~mL}$ and the average operation time was $192 \pm 126.59$ mins. Among the 35 patients undergoing infection mesh removal, 7 patients had mesh placement again and 28 patients did not. Drainage tube drainage and exudation were performed on 35 individuals with mesh infection.

\begin{tabular}{|ll|}
\hline TABLE2: operation statement \\
\hline Number \\
\hline Patch removal or not \\
Yes & 35 \\
No & 0 \\
\hline Intraoperative complication & 0 \\
Yes & 35 \\
No & \\
\hline Intraoperative account of blooding & $10.88 \pm 4.65$ \\
\hline Length of operation(min) & $192 \pm 126.59$ \\
\hline Surgical procedure & 19 \\
Open surgery & 16 \\
laparoscopy & \\
\hline Place the patch again & 7 \\
Yes & 28 \\
No & 35 \\
\hline Drainage & 0 \\
\hline Yes & \\
\hline
\end{tabular}

The patient's postoperative situation was unfolded in table3, The time of extubation in the all patients was $11.30 \pm 8.61$ days. A total of 35 patients were hospitalized for $23.74 \pm 15.91$ days. In 35 patients with patch infection, 1 patient was infected with Anaerococcus, 1 patients were infected with Enterococcus faecalis, 3 patients were infected with Escherichia coli, 2 patients were infected with Staphylococcus Epidermidis, 3 patients were infected with Pseudomonas aeruginosa, 1 patient was infected with Klebsiella pneumoniae, 3 patients were infected with mycobacterium fortuitum, 22 patients were infected with Staphylococcus aureus, 1 patient was infected with coagulase-negative staphylococcus, 1 patient 
was infected with Staphylococcus Aureus, Enterococcus Faecalis, anaerobic coccus. Of the 35 patients were given antibiotics for $19.83 \pm 22.10$ days.

\begin{tabular}{|ll|}
\hline TAB3: The patient's post-op status & \\
\hline Number & $11.30 \pm 8.61$ \\
\hline Time to pull out the drain(day) & 1 \\
\hline Bacterial type & 1 \\
Anaerococcus & 3 \\
Enterococcus faecalis & 2 \\
Escherichia coli & 3 \\
Epidermis Staphylococcus aureus & 1 \\
Copper-green pseudo-monocytobacteria & 3 \\
Creber bacteria with pneumonia & 22 \\
Mycobacterium fortuitum & 1 \\
Staphylococcus aureus & 1 \\
Coagulase negative staphylococci & \\
Staphylococcus aureus $\square$ Enterococcus faecalis $\square$ Escherichia coli & $23.74 \pm 15.91$ \\
\hline Days in hospital(day) & 34 \\
follow-up & 1 \\
Good & $19.83 \pm 22.10$ \\
\hline Bad & \\
\hline Days of antibiotic use(day) & 1 \\
\hline
\end{tabular}

All patients were followed for a period ranging from one to 35 months, with a median follow-up of 19 months. During the follow-up period of 35 patients, The patients all in peace after surgery, without postoperative related long-term complications or recurrence of ipsilateral inguinal hernia. One patient had a history of recurrent infection and was hospitalized two times in a row at nine-month intervals. The patient eventally recovered well after the second treatment at the end of a process, and the infection did not reoccur.

\section{Discussion}

Every year, approximately one million synthetic patches are used in herniorrhaphy.[13] Mesh infection is a major cause of inguinal hernia repair failure [14-15]. The results showed that the incidence of patch- 
related infection after tension-free repair of inguinal hernia was $0.6 \%-8.0 \%[16-18]$. The total infection rate of laparoscopic herniorrhaphy was $0.7 \%-2 \%$, which was related to the nonstandard aseptic operation, incomplete hemostasis, and contamination of the Mesh itself[19]. the infection rate of open herniorrhaphy was $6-10 \%$ [20-21]. The recurrence rate of delayed deep patch infection is less than $5 \%$ in patients undergoing patch removal[22]. Although recurrent hernias may be caused by current infection patch removal procedures. In addition, depending on the patient's circumstances, the patch may be applied during the operation to prevent hernia recurrence [23]. Although there have been reports of successful cases in which the patch has not been removed by negative pressure drainage of the infected focus, because the patch has already become infected, as a foreign body and continues to exist as a source of an infection deep down, negative pressure drainage or dressing change alone cannot clear the necrotic tissue completely. Therefore, surgical removal of the patch remains the most common treatment for patch infection.

The majority of patients in this research were moved from primary care institutions. With the popularity of hernia surgery, primary care hospitals have frequently performed tension-free repair of inguinal hernias, particularly the use of mesh plug repair hernia defect. The level of medical treatment, the type of patch used, the technique of operation, the concept of operation, and the instruments are closely related to the postoperative infection of the patch. According to the 2018 International Inguinal Hernia Guide, "The qualification and experience of the surgeon is an important factor in evaluating the outcome of inguinal hernia surgery." It is also important to understand the anatomy of the inguinal region and surrounding tissue next to the relationship, as well as the spermatic cord blood vessels, Vas deferens, ILIAC blood vessels, and other key tissue concentrations. The basic concept of tension-free repair of inguinal Hernia and the characteristics of patch materials are helpful to guarantee the curative effect and reduce postoperative complications[24]. In this study, 35 of the patients with delayed patch infection underwent patch removal and infection necrosis lesion removal. Note that the patch should be removed as completely as possible during the procedure, it may lead to the recurrence of infection, or even other serious complications, such as intestinal adhesions addition, patients with sinus tract formation were treated in this study with an intraoperative injection of methylene blue to completely excise the affected sinus tract, and debridement was performed according to the tracing range, followed by a series of rinses with hydrogen peroxide and normal saline containing iodophor to avoid residual infection. Due to the large surgical wound, more bleeding and oozing fluid in the wound during the operation, and the reinfection of the surgical site due to the easy multiplication of bacteria, it is necessary to pay attention to hemostasis and thorough removal of necrotic tissue in the surgical field during the operation, drainage tube or direct open wound drainage dressing to reduce the postoperative Hematoma and hematoma formation, to avoid postoperative wound infection lasting.

Early detection of infection usually occurs in patients with abdominal wall skin redness and swelling, pain, purulent discharge, and other local infection, after dressing change, drainage, empirical use of antibiotics and other conservative symptomatic treatment, if the wound cannot heal, can cause deep infection and patch infection, the performance of local abscess or chronic sinus formation. Besides all the symptoms and signs, ultrasound, CT, MRI, and sinus contrast testing can assess the size, length, 
depth, and course of the abscess chamber[25-27]. If the patient also has urinary system symptoms such as bladder irritation, cystoscopy is an option[28]. In addition, preoperative drainage fluid or wound secretion and intraoperative secretion from the infected area were collected for bacterial culture to reveal the type of bacterial infection. In this study, 35 patients were tested for bacterial culture, and most of the pathogens were Gram-positive bacteria (Staphylococcus aureus). The results are consistent with those reported in the literature[29-30]. At present, there is no consensus on the timing of surgical intervention. If the infection of the patch is clear, debridement should be performed as soon as possible. Delayed Mesh infections following tension-free herniorrhaphy are often more difficult to self-cure due to implants such as Mesh, so in this study, two patients were re-infected after debridement, and in one case, the residual patch was discovered during re-debridement and was completely controlled after two consecutive operations. It is a great challenge for surgeons to remove the patch as completely as possible while avoiding side effects. This is dependent on previous tension-free inguinal hernia repair employing materials, surgical methods, understanding of the anatomy of the inguinal area, and surgical evaluation of the scope of infection, and the determination of the scope of debridement[31].

Therefore, managing delayed patch infection after inguinal hernia repair is extremely challenging, and understanding how to prevent infection is critical. According to the 2018 international inguinal hernia guidelines, elective inguinal herniorrhaphy is a clean wound (class I incision) operation that does not normally involve the use of prophylactic antibiotics but is performed in high-risk populations, empiric prophylactic use of antibiotics (recommended to be administered intravenously 30-60 minutes before skin incision) can reduce the incidence of infection, the high-risk groups include The elderly, diabetes, obesity, emaciation, recurrent hernia, chemotherapy or radiotherapy and other immunocompromised inguinal hernia patients [32-33]. At the same time, for giant direct hernia patients, the incidence of postoperative Hematoma is generally high, in the process of aspiration can cause the secondary infection. Therefore, to reduce the incidence of Hematoma and effective treatment of Hematoma, to prevent postoperative inguinal hernia repair patch infection is of great significance. To avoid Mesh infection after inguinal hernia repair, it is necessary to take active and effective preventive measures during the perioperative period. Postoperative infection should be detected treated early appropriately, based on the patient's patch infection range, the cause of infection, as well as other factors, to develop an individual treatment plan. An infected patch usually needs to be removed surgically, so how to balance the "Protection of important structures such as the spermatic cord, Vas deferens, and ILIAC blood vessels" against the "Complete removal of the infected patch and necrotic tissue", it's still a work in progress.

Our preliminary study deemed that patch removal is the first choice for treatment of delayed patch infection after inguinal hernioplasty. It was safety and effectiveness. So we summarized four basic principles should be followed in the management of delayed Mesh infection after inguinal hernia repair: (1) timely operation; (2) complete and thorough removal of the Mesh as far as feasible; (3) avoiding injury to the large vessels in the surgical area, to avoid the formation of Hematomas; (4) timely drainage and infected tissue bacterial culture, and targeted use of antibiotics for treatment. Mesh removal, 
according to the above principles, is a safe and durable therapy for patients with delayed infection of Mesh after inguinal hernia repair.

\section{Declarations}

\section{Compliance with ethical standards}

Ethical approval.:The protocol was approved by the the Ethics Committee of Shaanxi Provincial People's Hospital.Human and animal rights As a retrospective review, no experimental conditions were implement. All human participants received adequate treatments, thus, no rights were violated. No animals were involved in this study.

Informed consent: Informed consent as a retrospective review, formal consent was not required.

Conflict of interest:All authors declare no conflicts of interest.

\section{Authors contributions}

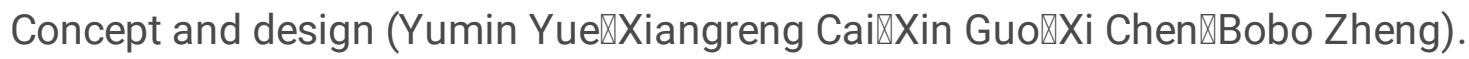

Procedures (Yumin Yue『Xiangreng CaiðXin Guo).

Writing of manuscript (Yumin Yue区Xiangreng Cai).

Manuscript corrections (Bobo Zheng, Xi Chen).

\section{Data availability}

The datasets generated and analysed during the current study are not publicly available due not public but are available from the corresponding author on reasonable request.

\section{References}

1. Delikoukos S, Tzovaras G, Liakou P, Mantzos F, Hatzitheofilou C. Late-onset deep mesh infection after inguinal hernia repair. Hernia. 2007 Feb;11(1):15-7. doi: 10.1007/s10029-006-0131-1. Epub 2006 Aug 29. (2007)

2. Lydeking L, Johansen N, Oehlenschläger J, Bay-Nielsen M, Bisgaard T. Re-recurrence and pain 12 years after laparoscopic transabdominal preperitoneal (TAPP) or Lichtenstein's repair for a recurrent inguinal hernia: a multi-centre single-blinded randomised clinical trial. Hernia. 2020 Aug;24(4):787792. doi: 10.1007/s10029-020-02139-0. Epub 2020 Feb 25. (2020)

3. Memon GA, Shah SKA, Habib-Ur-Rehman. An experience with mesh versus darn repair in inguinal hernias. Pak J Med Sci. 2017 May-Jun;33(3):699-702. doi: 10.12669/pjms.333.13257. (2017) 
4. Chen F, Liu M, Jin C, Wang F, Shen Y, Zhao F, Chen J. Tension-Free Mesh Repair for Incarcerated Groin Hernia: A Comparative Study. SurgInnov. 2020 Aug;27(4):352-357. doi:

10.1177/1553350620901392. Epub 2020 Jan 29. (2020)

5. Tolino MJ, Tripoloni DE.Late-onset deep mesh infection after inguinal hernia repair.Hernia. 2008 Feb;12(1):107; author reply 109. doi: 10.1007/s10029-007-0279-3. Epub 2007 Sep 13. (2008)

6. Brown RH, Subramanian A, Hwang CS, Chang S, Awad SS. Comparison of infectious complications with synthetic mesh in ventral hernia repair. Am J Surg. 2013 Feb;205(2):182-7. doi: 10.1016/j.amjsurg.2012.02.023. (2013)

7. Colbran R, Nicol A, Ng J. Delayed mesh infection presenting as an abdominal mass after laparoscopic inguinal hernia repair. ANZ J Surg. 2020 Oct;90(10):2127-2129. doi:

10.1111/ans.15742. Epub 2020 Feb 6. (2020)

8. Meagher $\mathrm{H}$, Clarke Moloney M, Grace PA. Conservative management of mesh-site infection in hernia repair surgery: a case series. Hernia. 2015 Apr;19(2):231-7. doi: 10.1007/s10029-013-1069-8. Epub 2013 Mar 16. (2015)

9. Luhmann A, Moses A. Successful conservative treatment of a candida albicans intraperitoneal mesh infection following laparoscopic ventral hernia repair. Hernia. 2015 Oct;19(5):845-7. doi: 10.1007/s10029-013-1183-7. Epub 2013 Nov 13. (2015) [10] Tolino MJ, Tripoloni DE, Ratto R, Garcia MI. Infections associated with prosthetic repairs of abdominal wall hernias: pathology, management and results. Hernia. 2009 Dec;13(6):631-7. doi: 10.1007/s10029-009-0541-y. (2009)

10. Luo Y, Wang $C$. The clinical characteristics and treatment of mesh infection after laparoscopic inguinal hernia repair: Two cases report and literature review. Asian J Surg. 2021 Aug 9:S10159584(21)00479-6. doi: 10.1016/j.asjsur.2021.07.062. Epub ahead of print. (2021)

11. Sakamoto T, Fujiogi M, Ishimaru M, Matsui H, Fushimi K, Yasunaga H. Comparison of postoperative infection after emergency inguinal hernia surgery with enterectomy between mesh repair and nonmesh repair: a national database analysis. Hernia.2021 Jun 17.doi: 10.1007/s10029-021-02439-z. Epub ahead of print. (2021)

12. van Veenendaal N, Simons M, Hope W, Tumtavitikul S, Bonjer J; HerniaSurge Group. Consensus on international guidelines for management of groin hernias.SurgEndosc. 2020 Jun;34(6):2359-2377. doi: 10.1007/s00464-020-07516-5. Epub 2020 Apr 6. Erratum in: SurgEndosc. 2020 Apr 22; (2020)

13. Wang D, Fu Y, Liu Q, Chen J. Maximal Infected Mesh Removal with Methylene Blue Injection for Mesh Infection after Inguinal Hernia Repair. Surg Infect (Larchmt). 2021 Apr;22(3):347-352. doi: 10.1089/sur.2020.133. Epub 2020 Aug 4. (2021)

14. O'Brien WJ, Ramos RD, Gupta K, Itani KMF. Neural Network Model to Detect Long-Term Skin and Soft Tissue Infection after Hernia Repair.Surg Infect (Larchmt). 2021 Sep;22(7):668-674. doi: 10.1089/sur.2020.354. Epub 2020 Dec 1. (2020)

15. Mazaki T, Mado K, Masuda H, Shiono M, Tochikura N, Kaburagi M. A randomized trial of antibiotic prophylaxis for the prevention of surgical site infection after open mesh-plug hernia repair. Am J Surg. 2014 Apr;207(4):476-84. doi: 10.1016/j.amjsurg.2013.01.047. (2014) 
16. Gachabayov M, Latifi R. Different etiopathogeneses in early-onset and late-onset inguinal hernia mesh infections in a prospectively evaluated cohort. ActaChir Belg. 2021 Jun;121(3):164-169. doi: 10.1080/00015458.2019.1689648. Epub 2019 Nov 11. (2021)

17. Liu J, Zhai Z, Chen J. The Use of Prosthetic Mesh in the Emergency Management of Acute Incarcerated Inguinal Hernias.SurgInnov. 2019 Jun;26(3):344-349. doi: 10.1177/1553350619828900. Epub 2019 Feb 8. (2019)

18. Perez AR, Roxas MF, Hilvano SS. A randomized, double-blind, placebo-controlled trial to determine effectiveness of antibiotic prophylaxis for tension-free mesh herniorrhaphy. J Am Coll Surg. 2005 Mar;200(3):393-7; discussion 397-8. doi: 10.1016/j.jamcollsurg.2004.10.037. (2005)

19. Pokala B, Armijo PR, Flores L, Hennings D, Oleynikov D. Minimally invasive inguinal hernia repair is superior to open: a national database review. Hernia. 2019 Jun;23(3):593-599. doi: 10.1007/s10029019-01934-8. Epub 2019 May 9. (2019)

20. Wu JJ, Way JA, Eslick GD, Cox MR. Transabdominal Pre-Peritoneal Versus Open Repair for Primary Unilateral Inguinal Hernia: A Meta-analysis. World J Surg. 2018 May;42(5):1304-1311. doi: 10.1007/s00268-017-4288-9. (2018)

21. Rehman S, Khan S, Pervaiz A, Perry EP. Recurrence of inguinal herniae following removal of infected prosthetic meshes: a review of the literature. Hernia. 2012 Apr;16(2):123-6. doi: 10.1007/s10029-0110873-2. Epub 2011 Aug 20. (2011) [23]Delikoukos S, Tzovaras G, Liakou P, Mantzos F, Hatzitheofilou C. Late-onset deep mesh infection after inguinal hernia repair. Hernia. 2007 Feb;11(1):15-7. doi: 10.1007/s10029-006-0131-1. Epub 2006 Aug 29. (2007)

22. HerniaSurge Group. International guidelines for groin hernia management.Hernia. 2018 Feb;22(1):1165. doi: 10.1007/s10029-017-1668-x. Epub 2018 Jan 12. (2018)

23. Li Destri G, Cocuzza A, Cavallaro M. Late cutaneous fistula after inguinal hernia repair without prosthesis. Acta Chir Belg. 2010 Nov-Dec;110(6):609-10.(2010)

24. Stommel M, van der Velden AP, Wijnen R, Wijnen M. Laparoscopic diagnosis and treatment of an enterocutaneous fistula after inguinal hernia correction. Eur J Pediatr Surg. 2010 Sep;20(5):341. doi: 10.1055/s-0029-1243629. Epub 2010 Oct 25. Erratum in: Eur J Pediatr Surg. 2010 Sep;20(5):e2. van der Velden, A S [corrected to van der Velden, A P Schouten]. (2010)

25. Jayamanne $H$, Williams GL, Stephenson BM. Colocutaneous fistula after left inguinal hernia repair using the mesh plug technique. Surgery. 2009 Jun;145(6):690. doi: 10.1016/j.surg.2009.03.013. (2009)

26. Manikandan R, Burke Y, Srirangam SJ, Collins GN. Vesicocutaneous fistula: an unusual complication of inguinoscrotal hernia. Int J Urol. 2003 Dec;10(12):667-8. doi: 10.1046/j.1442-2042.2003.00715.x. (2003)

27. Celdrán A, Esteban J, Mañas J, Granizo JJ. Wound infections due to Mycobacterium fortuitum after polypropylene mesh inguinal hernia repair. J Hosp Infect. 2007 Aug;66(4):374-7. doi: 10.1016/j.jhin.2007.05.006. Epub 2007 Jul 25. (2007) 
28. Kathju S, Nistico L, Lasko LA, Stoodley P. Bacterial biofilm on monofilament suture and porcine xenograft after inguinal herniorrhaphy. FEMS Immunol Med Microbiol. 2010 Aug;59(3):405-9. doi: 10.1111/j.1574-695X.2010.00691.x. Epub 2010 Apr 29. (2010)

29. Wada Y, Nishi M, Yoshikawa K, Higashijima J, Miyatani T, Tokunaga T, Takasu C, Kashihara H, Ishikawa D, Yoshimoto T, Shimada M. Usefulness of virtual three-dimensional image analysis in inguinal hernia as an educational tool. SurgEndosc. 2020 May;34(5):1923-1928. doi: 10.1007/s00464-019-06964-y. Epub 2019 Jul 16. (2020) [32] Erdas E, Medas F, Pisano G, Nicolosi A, Calò PG. Antibiotic prophylaxis for open mesh repair of groin hernia: systematic review and metaanalysis. Hernia. 2016 Dec;20(6):765-776. doi: 10.1007/s10029-016-1536-0. Epub 2016 Sep 3. (2016)

30. Zhuo Y, Zhang Q, Tang D, Cai D. The effectiveness of i.v. cefuroxime prophylaxis of surgical site infection after elective inguinal hernia repair with mesh: A retrospective observational study. Eur $\mathrm{J}$ ClinPharmacol. 2016 Sep;72(9):1033-9. doi: 10.1007/s00228-016-2067-4. Epub 2016 May 10. (2016) 